data collection, LP opening pressure $<20 \mathrm{~cm}$ of water or secondary causes for IIH.

Results 45 cases identified; 30 within the Greater Hobart region, population of 229,088 (June 2016-June 2017). Cumulative incidence was $6.55 / 100,000$ (incidence rate $0.06 / 1000$ ) with classification based on Neurologist diagnosis and 5.46/ $100,000(0.05 / 1000)$ with classification according to MDC. $100 \%$ of the cohort were female. Mean age was 26.7 (range $17-45$ ) and mean weight was $105.3 \mathrm{~kg}$ (range 78-170). Headache was the most commonly reported symptom. $8.9 \%(4 / 45)$ of the total cohort were medically refractory (requiring VP/LP shunting). Ophthalmology services initiated 51\% (23/45) of the referrals.

Conclusions Our incidence rates are higher than rates in previous studies for population subsets of young women.

\section{LYMPHOMA: A GREAT IMITATOR IN NEUROLOGY AND ITS MANY FACES}

${ }^{1}$ Wei Z Yeh*, 'Subramanian Muthusamy, ${ }^{2}$ Penny McKelvie, ${ }^{1}$ Steven Collins, ${ }^{1}$ Ann French, ${ }^{3}$ Robin Filshie, 'Katrina Reardon. 'Department of Neurology, St Vincent's Hospital Melbourne, Fitzroy, VIC, Australia; 'Department of Pathology, St Vincent's Hospital Melbourne, Fitzroy, VIC, Australia; ${ }^{3}$ Department of Haematology, St Vincent's Hospital Melbourne, Fitzroy, VIC, Australia

\subsection{6/jnnp-2019-anzan.69}

Introduction The label 'great imitator' refers to conditions which can cause varied manifestations and mimic diseases. Lymphoma is worthy of this title. We present three cases.

Cases 1: 66-year-old-man with progressive vertical diplopia and unsteady gait over four weeks. MRI brain and spine demonstrated a supratentorial para-falcine soft tissue lesion, mid-thoracic cord enhancement and right axillary mass. Serum ACE was elevated. Serum HIV serology was positive. Right axillary mass core biopsy diagnosed Burkitt lymphoma.

2: 50-year-old man with a 4-week history of constitutional symptoms on a background of ITP and splenomegaly. During admission he developed urinary retention, bilateral lower limb weakness and numbness and confusion. Infectious and vasculitic screens were unremarkable. CT chest, abdomen and pelvis demonstrated splenomegaly. CSF and bone marrow analyses were non-diagnostic. A random skin biopsy diagnosed intravascular lymphoma.

3: 65-year-old man with two weeks of headache and diplopia on a background of previously treated Burkitt lymphoma. CSF analysis showed $45 \times 10^{6} / \mathrm{L}$ white cells and glucose $<0.6 \mathrm{mmol} / \mathrm{L}$. Cytologic analysis was negative for malignancy. Bacterial culture and Cryptococcal antigen were negative. FDG-PET dramatically showed disseminated spinal and cranial leptomeningeal disease. MRI brain showed thin dural thickening correlating to area of increased uptake on PET. He was diagnosed with Burkitt lymphoma relapse and treated with chemotherapy and autologous stem cell transplant.

Conclusion The varied manifestations in our cases demonstrate the ability for lymphoma to mimic infective, inflammatory, granulomatous (including sarcoidosis) and neoplastic aetiologies. When the diagnosis is uncertain, the possibility of this great imitator should be considered.

\section{THE GASTROINTESTINAL MICROBIOME IN PARKINSON'S DISEASE: IMPACTS OF MOTOR AND NON-MOTOR FEATURES, MEDICATIONS, LIFESTYLE AND DIET}

${ }^{1}$ Michal Lubomski*, ${ }^{2}$ Ai Huey Tan, ${ }^{2}$ Shen-Yang Lim, ${ }^{3}$ Andrew Holmes, ${ }^{1}$ Ryan L Davis, ${ }^{1}$ Carolyn M Sue. ${ }^{1}$ Department of Neurogenetics, Kolling Institute, University of Sydney and Royal North Shore Hospital, Sydney, NSW, Australia; ${ }^{2}$ Neurology Department, Mah Pooi Soo and Tan Chin Nam Centre for Parkinson's and Related Disorders, Faculty of Medicine, University of Malaya, Kuala Lumpur, Malaysia; ${ }^{3}$ School of Life and Environmental Sciences, The Charles Perkins Centre, University of Sydney, Sydney, NSW, Australia

\subsection{6/jnnp-2019-anzan.70}

Introduction The human gastrointestinal microbiome (GM) has been proposed to be integral in the pathogenesis of Parkinson's disease (PD). Evidence supports a bidirectional interaction between the brain and the gut that is mediated by the GM. Dysbiosis of the GM is believed to negatively influence vital physiological functions in many diseases.

Methods We reviewed the literature on changes in human physiological function associated with gut microbial community states in PD. In particular, we evaluated the literature for effects of GM dysbiosis on motor and non-motor features, dietary and lifestyle factors and medication use in PD.

Results Altered GM profiles in PD have been suggested to disrupt vital signalling pathways within the microbiota-gutbrain axis, integral to regulating physiological digestive function and metabolic homeostasis. Unfavourable variations in the GM have been shown to perturb mood (anxiety/ depression), cognition, perception (hallucinations/delusions), gastrointestinal motility, including constipation in PD. Further, varied clinical motor phenotypes, including postural instability and gait disturbance have been attributed to alterations in the GM, in addition to the use of catechol-Omethyltransferase inhibitors, anticholinergics and levodopa. Variations in dietary and lifestyle factors have also been inferred to cause alterations in GM profiles, including caffeine consumption, macronutrient intake, smoking and the effects of ageing and exercise.

Conclusions It is apparent from the mounting evidence that alterations in the GM are intimately involved in PD pathogenesis. However, the GM can also be modulated by dietary, lifestyle and treatment factors that may influence motor and nonmotor features as well as disease progression.

\section{NOVEL WIRELESS S - PATCH DEVICE MAY EFFICIENTLY DETECT ATRIAL FIBRILLATION IN PATIENTS WITH ISCHAEMIC STROKE}

${ }^{1}$ Suzana Lazarovska* ${ }^{1} \mathrm{Hui}$ Tie, ${ }^{1}$ Andrew Hopkins, ${ }^{1}$ Hany Dimitri, ${ }^{2}$ Upul Premawardhana, ${ }^{3}$ Alan Mcdougall, ${ }^{4}$ Sumana Gopinath, ${ }^{5}$ Josephine Chow, ${ }^{1}$ Rohan Rajaratnam. ${ }^{1}$ Cardiology, Liverpool Hospital, Sydney, NSW, Australia; ${ }^{2}$ Cardiology, Campbelltown Hospital, Sydney, NSW, Australia; ${ }^{3}$ Neurology, Liverpool Hospital, Sydney, NSW, Australia; ${ }^{4}$ Neurology, Campbelltown Hospital, Sydney, NSW, Australia; ${ }^{5}$ Liverpool Renal Clinical Research Centre, Co-Director, Sydney, NSW, Australia

\subsection{6/jnnp-2019-anzan.71}

Introduction Atrial fibrillation (AF) is the most common atrial arrhythmia leading to increased risk of thromboembolic events. Ward telemetry (WT) has a low detection rate for AF in stroke patients and often patients are not appropriately treated with anticoagulation. This study compares conventional 2-day WT versus 4 -day wireless $S$-patch monitoring to detect AF. 